\title{
Three-phase power transformer modelling in AC/DC traction substations
}

\author{
Cornelia A. Bulucea ${ }^{1, *}$, Doru A. Nicola ${ }^{1}$, Nikos E. Mastorakis ${ }^{2}$, and Carmen A. Bulucea ${ }^{3}$ \\ ${ }^{1}$ University of Craiova, Faculty of Electrical Engineering, Craiova 200440, Romania \\ ${ }^{2}$ Technical University of Sofia, English Language Faculty of Engineering, Sofia 1000, Bulgaria \\ ${ }^{3}$ University of Medicine and Pharmacy of Craiova, Faculty of Medicine, Craiova 200349, Romania
}

\begin{abstract}
Three-phase power transformer is one of the most important elements in the electric power systems, and it plays a significant role in terms of energy savings. Since the efficiency standards can be expressed in terms of electrical efficiency, in an attempt to improve the transformer efficiency, in this study an enhancement of three-phase power transformer modelling with space phasors is presented. There are established the equations with space phasors of the three-phase transformer with symmetrical compact core. This equations system can be used to analyze the dynamic regimes of three-phase transformers. In this paper have been analyzed some aspects of three-phase power transformer operation in a AC/DC traction substation.
\end{abstract}

\section{Introduction}

Nowadays, because of economic and business growth, standards of life and development of civilization are too often interpreted in correlation with the use of electricity, and the demand of electricity is constantly rising. Threephase power transformer is one of the most important elements in the electric power systems, and it plays a significant role in terms of energy savings [1-4]. The transmission and distribution of electricity through different voltage levels are possible due to the use of power transformers. The efficiency and sustainability of power transformers are in correlation with the reliability of the whole network, and could have considerable economic and environmental impact.

Forecast based on mathematical models enlarges our beliefs on the world functionality [5]. Although the mathematical modeling is a complex process and entails a large element of compromise the interacting systems in the real world can be studied identifying the most important interrelations of the systems [2-7]. Since the efficiency standards can be expressed in terms of electrical efficiency depending on load characteristics, in an attempt to improve the transformer efficiency, below an enhancement of three-phase power transformer modelling with space phasors is presented [3-4].

\section{Three-phase transformer modelling with space phasors}

Basically, three-phase transformers are widely used since three phase power is the common way to produce, transmit and use the electrical energy. A three-phase transformer

\footnotetext{
* Corresponding author: abulucea@em.ucv.ro
}

transfers electric power from the three-phase primary winding through inductively coupled three-phase secondary winding, changing values of three-phase RMS voltage and current [3-4]. Most common, the transformers windings are wound around a ferromagnetic core.

In this study we take into consideration a three-phase transformer with a non-saturated magnetic core, and in a symmetrical construction, as depicted in Fig.1.
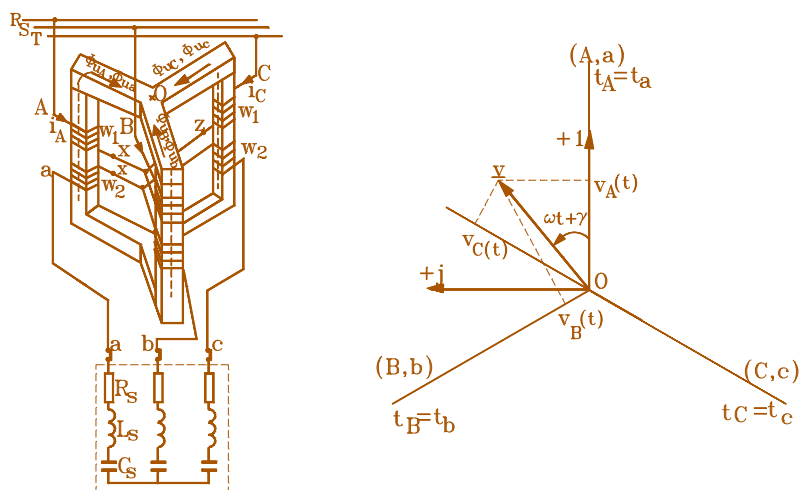

Fig.1. Three-phase power transformer

The primary phase windings $(A-X),(B-Y)$ and $(C-Z)$ are identical, each of them having $w_{l}$ turns and the electric resistance $R_{l}$. Similarly, the secondary phase windings ( $a$ $x$ ), (b-y) and (c-z) are identical each of them having $w_{2}$ turns and the electric resistance $R_{2}$. Moreover, the threephase primary winding is connected in star $(\mathrm{Y})$ being supplied by the RST power network, while the three-phase secondary winding is connected in star (y) and is supplying the three-phase load connected in star, with the parameters $R_{s}-L_{s}-C_{s}$ on each phase, as shown in Fig.1. 
Varying currents flowing in the primary winding (due to the varying phase voltages $u_{A}, u_{B}$ and $u_{C}$ ) create a varying magnetic flux in the transformer core, and thus a varying magnetic field through the secondary winding. This varying magnetic field induces a varying electromotive force in the secondary winding. Since a three-phase electric load is connected to the secondary winding, electrical energy will be transferred from the primary circuit through the transformer to the load [1-4, 6-7].

In this paper the three-phase electromagnetic phenomena will be described into the space phasors theory [3-4,8].

In case of three-phase transformer all variables $\left(\underline{u}_{1}=\right.$ primary voltage; $\underline{i}_{1}=$ primary current; $\underline{u}_{2}=$ secondary voltage and $\underline{i}_{2}=$ secondary current) are not real but complex mathematical quantities. In this context, in the study of the three-phase transformer one can use the space phasors method, highlighting that the time axes $t_{A}=t_{a}, t_{B}=t_{b}$ and $t_{C}=t_{c}$ are physically associated at the axes of the threephase primary and secondary windings, which are symmetrically disposed in space.

In this framework we obtain the voltage equations of the primary phase windings as follows:

$$
\begin{gathered}
u_{A}=R_{1} \cdot i_{A}+\frac{d}{d t} \Psi_{A} \\
u_{B}=R_{1} \cdot i_{B}+\frac{d}{d t} \Psi_{B} \\
u_{C}=R_{1} \cdot i_{C}+\frac{d}{d t} \Psi_{C}
\end{gathered}
$$

By amplifying the equations (1) with $2 / 3,2 a / 3,2 a^{2} / 3$ and subsequently summing them will result the equation with space phasors as below:

$$
\underline{u}_{1}=R_{1} \cdot \underline{i}_{1}+\frac{d}{d t} \underline{\Psi}_{1}
$$

where:

$$
\begin{gathered}
\underline{u}_{1}=\frac{2}{3} \cdot\left(u_{A}+a \cdot u_{B}+a^{2} \cdot u_{C}\right) \\
\underline{i}_{1}=\frac{2}{3} \cdot\left(i_{A}+a \cdot i_{B}+a^{2} \cdot i_{C}\right) \\
\underline{\Psi}_{1}=\frac{2}{3} \cdot\left(\Psi_{A}+a \cdot \Psi_{B}+a^{2} \cdot \Psi_{C}\right)
\end{gathered}
$$

are the space phasors of voltages $\underline{u}_{1}$, currents $\underline{i}_{1}$ and total fluxes $\underline{\Psi}_{l}$ corresponding to primary phase windings of three-phase transformer.

Similarly, we obtain the voltage equations of the secondary phase windings as below:

$$
\begin{gathered}
u_{a}=-R_{2} \cdot i_{a}-\frac{d}{d t} \Psi_{a} \\
u_{b}=-R_{2} \cdot i_{b}-\frac{d}{d t} \Psi_{b} \\
u_{c}=-R_{2} \cdot i_{c}-\frac{d}{d t} \Psi_{c}
\end{gathered}
$$

By amplifying the equations (4) with $2 / 3,2 a / 3,2 a^{2} / 3$ and subsequently summing them will result the voltage equation with space phasors as follows:

$$
\underline{u}_{2}=-R_{1} \cdot \underline{i}_{2}-\frac{d}{d t} \underline{\Psi}_{2}
$$

In equation (5) $\underline{u}_{2}, \underline{i}_{2}$ and $\underline{\Psi}_{2}$ denote, respectively, the space phasors of voltages, currents and fluxes, corresponding to secondary phase windings of three-phase transformer.

One could note that the symmetrical three-phase transformer with compact ferromagnetic core has the phase windings magnetically coupled. Consequently, the total magnetic fluxes will be determined based on superposition principle. As example below there are presented the relationships for the fluxes through the total turns surface of windings $\mathrm{A}-\mathrm{X}$ and $\mathrm{a}-\mathrm{x}$ that are wound around the same column of ferromagnetic core.

$$
\begin{aligned}
& \Psi_{A}=\Psi_{\sigma A}+\Psi_{u A}+\Psi_{B A}+\Psi_{C A}+\Psi_{a A}+\Psi_{b A}+\Psi_{c A} \\
& \Psi_{a}=\Psi_{\sigma a}+\Psi_{u a}+\Psi_{b a}+\Psi_{c a}+\Psi_{A a}+\Psi_{B a}+\Psi_{C a}
\end{aligned}
$$

Taking into consideration the magnetic core symmetry and in correlation with the positive sense of useful fascicular fluxes the relationships for the total magnetic coupling fluxes result as below:

$$
\begin{aligned}
& \Psi_{B A}=-\frac{1}{2} \cdot \Psi_{u B} ; \Psi_{C A}=-\frac{1}{2} \cdot \Psi_{u C} \\
& \Psi_{a A}=w_{1} \cdot \frac{\Psi_{u a}}{w_{2}} ; \Psi_{b A}=w_{1}\left(-\frac{1}{2} \cdot \frac{\Psi_{u b}}{w_{2}}\right) ; \Psi_{c A}=w_{1}\left(-\frac{1}{2} \cdot \frac{\Psi_{u c}}{w_{2}}\right) \\
& \Psi_{b a}=-\frac{1}{2} \cdot \Psi_{u b} ; \Psi_{C c a}=-\frac{1}{2} \cdot \Psi_{u c} \\
& \Psi_{A a}=w_{2} \cdot \frac{\Psi_{u A}}{w_{1}} ; \Psi_{B a}=w_{2}\left(-\frac{1}{2} \cdot \frac{\Psi_{u B}}{w_{1}}\right) ; \Psi_{C a}=w_{2}\left(-\frac{1}{2} \cdot \frac{\Psi_{u C}}{w_{1}}\right)
\end{aligned}
$$

Subsequently the expressions (6) can be rewritten as:

$$
\begin{aligned}
& \Psi_{A}=\Psi_{\sigma A}+\Psi_{u A}-\frac{1}{2}\left(\Psi_{u B}+\Psi_{u C}\right)+\frac{w_{1}}{w_{2}}\left[\Psi_{u a}-\frac{1}{2}\left(\Psi_{u b}+\Psi_{u c}\right)\right] \\
& \Psi_{a}=\Psi_{\sigma a}+\Psi_{u a}-\frac{1}{2}\left(\Psi_{u b}+\Psi_{u c}\right)+\frac{w_{1}}{w_{2}}\left[\Psi_{u A}-\frac{1}{2}\left(\Psi_{u B}+\Psi_{u C}\right)\right]
\end{aligned}
$$

Since the useful fascicular fluxes verify the equations:

$$
\Phi_{u A}+\Phi_{u B}+\Phi_{u C}=0 ; \Phi_{u a}+\Phi_{u b}+\Phi_{u c}=0
$$

by amplifying with the turn numbers will result the relations for the total useful magnetic fluxes as follows:

$$
\Psi_{u B}+\Psi_{u C}=-\Psi_{u A} ; \Psi_{u b}+\Psi_{u c}=\Psi_{u a}
$$

Based on expressions (10) the relationships (8) will become:

$$
\begin{aligned}
& \Psi_{A}=\Psi_{\sigma A}+\frac{3}{2} \cdot \Psi_{u A}+\frac{w_{1}}{w_{2}} \cdot \frac{3}{2} \cdot \Psi_{u a} \\
& \Psi_{a}=\Psi_{\sigma a}+\frac{3}{2} \Psi_{u a}+\frac{w_{1}}{w_{2}} \cdot \frac{3}{2} \cdot \Psi_{u A}
\end{aligned}
$$

One could note that in the case of three-phase compact core, due to the windings' magnetic coupling the useful fascicular flux corresponding to each phase winding is increased by $3 / 2$ in comparison with the ferromagnetic core with free fluxes.

$$
\text { Since } \Psi_{u A}=w_{1} \cdot \phi_{u A} \text {, respectively } \Psi_{u a}=w_{2} \cdot \phi_{u a} \text {, }
$$


and

$$
\phi_{u A}=\frac{w_{1} \cdot i_{A}}{R_{m u}}=w_{1} \cdot i_{A} \cdot \Lambda_{u} \quad \text { respectively }
$$

$\phi_{u a}=\frac{w_{2} \cdot i_{a}}{R_{m u}}=w_{21} \cdot i_{a} \cdot \Lambda_{u} \quad$ will result the relationships for the total magnetic fluxes $\Psi_{A}$ and $\Psi_{a}$ as below:

$\Psi_{A}=\Psi_{\sigma A}+\left(w_{1} \cdot i_{A}+w_{2} i_{a}\right) \cdot \frac{3}{2} \cdot w_{1} \cdot \Lambda_{u}$

$\Psi_{a}=\Psi_{\sigma u}+\frac{w_{2}}{w_{1}} \cdot\left(w_{1} \cdot i_{A}+w_{2} \cdot i_{a}\right) \cdot \frac{3}{2} \cdot w_{1} \cdot \Lambda_{u}$

Further one could remind that:

$$
\theta_{A \mu}=w_{1} \cdot i_{A}+w_{2} \cdot i_{a}=w_{1} \cdot i_{A \mu}
$$

and: $L_{u 1}=w_{1}^{2} \cdot \Lambda_{u}$

Consequently, the fluxes relationships (12) can be expressed as follows:

$$
\begin{aligned}
& \Psi_{A}=L_{\sigma 1} \cdot i_{A}+\frac{3}{2} \cdot L_{u 1} \cdot i_{A \mu} \\
& \Psi_{a}=L_{\sigma 2} \cdot i_{a}+\frac{w_{2}}{w_{1}} \cdot \frac{3}{2} \cdot L_{u 1} \cdot i_{A \mu}
\end{aligned}
$$

$$
\text { in which: } i_{A \mu}=i_{A}+\frac{w_{2}}{w_{1}} \cdot i_{a}
$$

Due to the symmetrical construction for the other two windings pairs ( $\mathrm{B}$ and $\mathrm{b}$, respectively $\mathrm{C}$ and $\mathrm{c}$ ) can be written the following equations:

$$
\begin{aligned}
& \Psi_{B}=L_{\sigma 1} \cdot i_{B}+\frac{3}{2} \cdot L_{u 1} \cdot i_{B \mu} ; \\
& \Psi_{b}=L_{\sigma 2} \cdot i_{b}+\frac{w_{2}}{w_{1}} \cdot \frac{3}{2} \cdot L_{u 1} \cdot i_{B \mu} ; \\
& \Psi_{C}=L_{\sigma 1} \cdot i_{C}+\frac{3}{2} L_{u 1} \cdot i_{C \mu} \\
& \Psi_{c}=L_{\sigma 2} \cdot i_{c}+\frac{w_{2}}{w_{1}} \cdot \frac{3}{2} \cdot L_{u 1} \cdot i_{C \mu} \\
& \text { in which: } i_{B \mu}=i_{B}+\frac{w_{2}}{w_{1}} \cdot i_{b} \\
& \text { respectively } i_{C \mu}=i_{C}+\frac{w_{2}}{w_{1}} \cdot i_{c}
\end{aligned}
$$

Subsequently, based on relationships (13) and (14) one could build the space phasors of magnetic fluxes of threephase transformer as below:

$$
\begin{aligned}
& \underline{\Psi}_{1}=\frac{2}{3} \cdot\left(\Psi_{A}+a \cdot \Psi_{B}+a^{2} \cdot \Psi_{C}\right)=L_{\sigma 1} \cdot \underline{i}_{1}+\underline{\Psi}_{u 1} \\
& \underline{\Psi}_{2}=\frac{2}{3} \cdot\left(\Psi_{a}+a \cdot \Psi_{b}+a^{2} \cdot \Psi_{c}\right)=L_{\sigma 2} \cdot \underline{i}_{2}+\underline{\Psi}_{u 2}
\end{aligned}
$$

In relationships (15) have been introduced the notations:

$$
\begin{aligned}
& \underline{\Psi}_{u 1}=L \cdot \underline{i}_{1 \mu} ; \underline{\Psi}_{u 2}=\frac{w_{2}}{w_{1}} \cdot L \cdot \underline{i}_{1 \mu} \\
& \underline{i}_{1 \mu}=\frac{2}{3} \cdot\left(i_{A \mu}+a \cdot i_{B \mu}+a^{2} \cdot i_{C \mu}\right)=\underline{i}_{1}+\frac{w_{2}}{w_{1}} \cdot \underline{i}_{2}
\end{aligned}
$$

where: $L=\frac{3}{2} L_{u 1}$ denotes the cyclical inductance of primary and $\underline{i}_{1 \mu}$ denotes the space phasor of magnetization currents.

With respect to the three-phase load connected at the transformer secondary terminals, the phase voltage equations are as follows:

$$
\begin{aligned}
& u_{a}=R_{s} \cdot i_{a}+L_{s} \cdot \frac{d i_{a}}{d t}+\frac{1}{C_{s}} \cdot \int i_{a} d t \\
& u_{b}=R_{s} \cdot i_{b}+L_{s} \cdot \frac{d i_{b}}{d t}+\frac{1}{C_{s}} \cdot \int i_{b} d t \\
& u_{c}=R_{s} \cdot i_{c}+L_{s} \cdot \frac{d i_{c}}{d t}+\frac{1}{C_{s}} \cdot \int i_{c} d t
\end{aligned}
$$

By amplifying the equations (17) with $2 / 3,2 a / 3,2 a^{2} / 3$ respectively, and subsequently summing them will result the voltage equation with space phasors of the three-phase load circuit, as below:

$\underline{u}_{2}=R_{s} \underline{i}_{2}+L_{s} \cdot \frac{d}{d t} \underline{i}_{2}+\frac{1}{C_{s}} \cdot \int \underline{i}_{2} d t$

Subsequently, by introducing the space phasors of the electromotive forces induced in primary and secondary windings, the equations of the three-phase transformer with symmetrical compact core can be ordered in the following space phasors system:

$$
\begin{aligned}
& \underline{u}_{1}=-\underline{e}_{1}+R_{1} \cdot \underline{i}_{1}+L_{\sigma 1} \cdot \frac{d}{d t} \underline{i}_{1} \\
& \underline{u}_{2}=\underline{e}_{2}-R_{2} \cdot \underline{i}_{2}-L_{\sigma 2} \cdot \frac{d}{d t} \underline{i}_{2} \\
& w_{1} \underline{i}_{1}+w_{2} \underline{i}_{2}=w_{1} \underline{i}_{1 \mu} \\
& \underline{e}_{1}=-\frac{d}{d t} \underline{\Psi}_{u 1} ; \underline{e}_{2}=-\frac{d}{d t} \underline{\Psi}_{u 2} \\
& \underline{\Psi}_{u 1}=L \cdot \underline{i}_{1 \mu} ; \underline{\Psi}_{u 2}=\frac{w_{2}}{w_{1}} \cdot \underline{\Psi}_{u 1}
\end{aligned}
$$

$L=\frac{3}{2} \cdot L_{u 1}$

$\underline{u}_{2}=R_{s} \cdot \underline{i}_{2}+L_{s} \cdot \frac{d}{d t} \underline{i}_{2}+\frac{1}{C_{s}} \cdot \int_{\underline{i}_{2}} d t$

Moreover, one could proceed to secondary reported to primary, with the secondary space phasors:

$$
\underline{i}_{2}^{\prime}=\frac{w_{2}}{w_{1}} \cdot \underline{i}_{2} ; \underline{u}_{2}^{\prime}=\frac{w_{1}}{w_{2}} \cdot \underline{u}_{2} ; R_{2}^{\prime}=\left(\frac{w_{1}}{w_{2}}\right)^{2} \cdot R_{2} ; L_{2}^{\prime}=\left(\frac{w_{1}}{w_{2}}\right)^{2} \cdot L_{2}
$$


further obtaining the equations of three-phase transformer with secondary reduced to primary:

$$
\begin{aligned}
& \underline{u}_{1}=-\underline{e}_{1}+R_{1} \cdot \underline{i}_{1}+L_{\sigma 1} \cdot \frac{d}{d t} \underline{i}_{1} \\
& \underline{u}_{2}=\underline{e}_{2}-R_{2}^{\prime} \cdot \underline{i}_{2}^{\prime}-L_{\sigma 2}{ }^{\prime} \cdot \frac{d}{d t} \underline{i}_{2}^{\prime} \\
& \underline{i}_{1}+\underline{i}^{\prime}=\underline{i}_{1 \mu} \\
& \underline{e}_{1}=\underline{e}_{2}{ }^{\prime}=-\frac{d}{d t} \underline{\Psi}_{u 1}, \underline{\Psi}_{u 1}^{\prime}=\underline{\Psi}_{u 2}=-L^{\prime} \cdot \underline{i}_{1 \mu} ;{ }^{\prime}=\frac{3}{2} L_{u 1} \\
& \underline{\Psi}^{\prime}=R_{s}^{\prime} \cdot \underline{i}_{2}^{\prime}+L_{s}{ }^{\prime} \cdot \frac{d}{d t} \underline{i}_{2}^{\prime}+\frac{1}{C_{s}^{\prime}} \cdot \int \underline{i}_{2}{ }^{\prime} d t
\end{aligned}
$$

The equations system (21) are on the whole conclusive. Particularly, in a permanent harmonic regime all space phasors of the three-phase symmetrical systems of sinusoidal quantities take the form $\underline{v}=S Q R T \underline{V} \cdot e^{j \omega t}$. Taking into account the space phasors derivation and integration relationships:

$$
\frac{d}{d t} \underline{v}=j \cdot \omega \cdot \underline{v} \quad \int \underline{v} d t=\frac{1}{j \cdot \omega} \cdot \underline{v}
$$

one could find the space phasors equations of system (21) rewritten in the classic form:

$$
\begin{aligned}
& \underline{u}_{1}=-\underline{e}_{1}+R_{1} \cdot \underline{i}_{1}+j \cdot X_{\sigma 1} \cdot \underline{i}_{1} \\
& \underline{u}_{2}{ }^{\prime}=\underline{e}_{2}{ }^{\prime}-R_{2}{ }^{\prime} \cdot \underline{i}_{2}{ }^{\prime}-j \cdot X_{\sigma 2} \cdot \underline{i}_{2}{ }^{\prime} \\
& \underline{i}_{1}+\underline{i}_{2}{ }^{\prime}=\underline{i}_{1 \mu}+\underline{i}_{10 a} ; \underline{i}_{10 a}=-\underline{e}_{1} / R_{F e} \\
& \underline{e}_{1}=\underline{e}_{2}{ }^{\prime}=-j \cdot \omega \cdot \underline{\Psi}_{u 1}=-j \cdot 3 / 2 \cdot X_{u 1} \cdot \underline{i}_{1 \mu} \\
& \underline{u}_{2}{ }^{\prime}=R_{s}{ }^{\prime} \cdot \underline{i}_{2}{ }^{\prime}+j X_{s} \cdot \underline{i}_{2}{ }^{\prime}
\end{aligned}
$$

One could emphasize that the equations system (21) can be used to analyze the dynamic regimes of three-phase transformers, being successfully applied, for instance, in the method of structural diagrams in analyzing the power transformer operation [8]. Subsequently we will analyze some aspects of three-phase power transformer operation in a $\mathrm{AC} / \mathrm{DC}$ traction substation.

\section{Power transformer in AC/DC traction substation}

The DC electrical traction substations are those fixed traction installations that receive electricity (in three-phase AC) from the national power system (at high voltage), reduce the voltage level and modify the current type (from $\mathrm{AC}$ to $\mathrm{DC}$ ) and, finally, distributes the electric power to contact line sections in order to supply the nonautonomous electric railway vehicles [9-11].
As spreading, the DC substations are used both in urban (surface and underground) electrical traction and in DC electrified railway traction. As a location, they are "indoor" installations, most of the equipment being arranged in a "cellular" structure (in sideboards).

The basics of the DC traction substations are the AC-DC conversion group.. Over time, the AC-DCconversion groups have made significant progress in terms of performance, efficiency, maintenance and reliability [8-9].

Nowadays, all substations are equipped with static rectifiers with diodes [9-11].

Optionally, reversible DC substations (with anti-parallel transformer- thyristorized inverter groups) can also be used to recover electrical energy in case of electric vehicle recuperative braking.

In principle, any DC traction substation consists of a high-voltage alternating current system (comprising: the three-phase primary line, three-phase high-voltage bars, the tripolar protective circuit breakers of the transformer rectifier group and the power transformers ) and a DC power system with $\mathrm{U}_{\mathrm{LC}}$ rated voltage (consisting of rectifier bridges, DC breakers and ultrafast DC switches).

The AC-DC conversion groups are made up of:

- a three phase transformer in order to reduce the actual voltage value (from $\mathrm{U}_{1}$ of the three-phase primary line to the $\mathrm{U}_{2}$ for supplying the rectifier) in close correlation with the continuous voltage $\mathrm{U}_{\mathrm{LC}}$ magnitude across the contact line, and

- a three-phase rectifier, usually with diodes (connected in three-phase bridge and mounted in "cabinets").

The basic structure of a rectifier system in a DC traction substation consists of a three-phase bridge of type Graetz bridge [9-10], depicted in Fig.2.

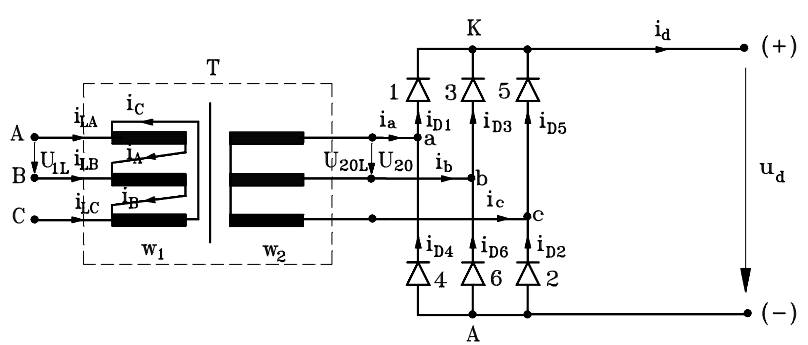

Fig.2. Rectifier with Graetz three-phase bridge

This bridge is powered from the secondary of a threephase transformer (T), usually with a Delta-Star (Dy) connection scheme, having the transformation ratio $\mathrm{K}$ (of the line voltages) given as follows:

$K=\frac{U_{I L}}{U_{20 L}}=\frac{1}{\sqrt{3}} \cdot \frac{w_{1}}{w_{2}}$

where:

$\mathrm{W}_{1}=$ the phase turns number of the primary winding (connected in $\Delta$ ), and

$\mathrm{w}_{2}=$ the phase turns number of the secondary winding (connected în star). 


\subsection{The rectified voltage (idealized)}

In order to study the idealized operation of the threephase rectifier bridge, the following hypotheses are accepted [9]:

1. The inductance $L_{d}$ (of the DC circuit) can be considered as infinitely high $\left(\mathrm{L}_{\mathrm{d}} \rightarrow \infty\right)$; consequently the DC current $i_{d}$ will be perfectly smooth, and constant over time $i_{d}=I_{d}$.

2. It is considered perfect magnetic coupling between the rectifier transformer windings. This means neglecting the transformer leakages $\left(\mathrm{L}_{\sigma 1} \rightarrow 0\right.$ and $\left.\mathrm{L}_{\sigma 2} \rightarrow 0\right)$, and consequently the neglect of the inductance $\mathrm{L}_{\mathrm{k}}$ of the swithching circuit $\left(\mathrm{L}_{k} \rightarrow 0\right)$. Therefore, sudden variations in currents are admitted, which is equivalent to neglecting the natural switching phenomenon.

3. There are neglected the ohmic resistance (primary $\mathrm{R}_{1} \rightarrow 0$ and secondary $\mathrm{R}_{2} \rightarrow 0$ ) of the rectifier transformer windings.

Under these conditions, the three-phase transformer $\mathrm{T}$ (fed into the primary) and seen on the secondary terminals will appear as a three phase (ideal) source with sinusoidal phase voltages $\mathrm{e}_{a} 0, \mathrm{e}_{\mathrm{b} 0}$ şi $\mathrm{e}_{\mathrm{c} 0}$ (symmetric, by direct sequence, with efffective values $\mathrm{E}_{20}$ ) so that the composed voltages (line voltages) can preserve their effective value $\mathrm{U}_{20 \mathrm{~L}}=\mathrm{U}_{20}$. Accordingly one can write:

$$
U_{20}=\frac{U_{1 L}}{K} ; \quad E_{20}=\frac{1}{\sqrt{3}} U_{20}
$$

If the three-phase (diode) bridge is fed from the ideal three-phase source (equivalent to the transformer) with the symmetrical sinusoidal phase voltages:

$$
\begin{aligned}
& e_{a 0}=\sqrt{2} E_{20} \sin \omega t \\
& e_{b 0}=\sqrt{2} E_{20} \sin \left(\omega t-\frac{2 \pi}{3}\right) \\
& e_{c 0}=\sqrt{2} E_{20} \sin \left(\omega t-\frac{4 \pi}{3}\right)
\end{aligned}
$$

then, at any time moment $\omega \mathrm{t}>0$, there will be only two diodes in conduction, namely:

a) only the diode in the cathodic group $(1,3,5)$ with the anode connected to that phase of the source with the highest positive instantaneous phase voltaget, and

b) only the diode in the anode group $(2,4,6)$ with the cathode connected to that phase of the source with the lowest negative instantaneous phase voltage.

All other diodes being momentarily subjected to inverse voltages are locked.

As an example, with the temporary origin $\omega \mathrm{t}=0$ (in the phase voltages diagram) when $\mathrm{e}_{\mathrm{c} 0}=\mathrm{e}_{\mathrm{a} 0}$ (see Fig.4, pos.a), left) it is noted that in the interval $[0, \pi / 3]$ only lead diodes 1 and 6 and the rectified voltage $u_{d}$ results as:

$$
u_{d}=e_{a 0}-e_{b 0}-2 \cdot U D=u_{a b^{-2}} \cdot U_{D}
$$

Here $u_{a b}=e_{a 0}-e_{b 0}$ represents the line voltage, and $U_{D}$ is the direct voltage drop at the terminals of any diode in conduction.

The situation analyzed above is repeated (with other pairs of diodes) six times in each $\mathrm{T}$ period. The sequence of conduction intervals of the three-phase bridge diodes is shown in Fig.3, pos.b). In addition, if direct voltage drops are also neglected on diodes temporarily in conduction (meaning if $U_{D} \rightarrow 0$ ), then the rectified voltage $u_{d}$ will be given (in each period $\mathrm{T}=1 / \mathrm{f}$ ) only by the ,positive elevations" of line voltages, exactly as is depicted in Fig.3, pos.c) (in left side, where the thickened curve represents the diagram $\left.\mathrm{u}_{\mathrm{d}}=\mathrm{f}(\omega \mathrm{t})\right)$.

Consequently, the rectified voltage $u_{d}$ is not constant over time (since it has $\mathrm{p}=6$ "elevations") but is is periodical, with the main period $\mathrm{T}_{1}=\mathrm{T} / \mathrm{p}$ or, in angular magnitude, with the angular period $\beta_{1}$ as below:

$\beta_{1}=\frac{2 \pi}{p}=\frac{2 \pi}{s \cdot q}=\frac{\beta}{s}=\frac{\pi}{3}$

The average value $U_{d 0}$ of the rectified voltage $u_{d}$ calculated on the inteval of a main period $\beta_{1}$ when the voltage $u_{d} \approx u_{b c}$ (see Fig.3, pos.c)) has the analytical expression:

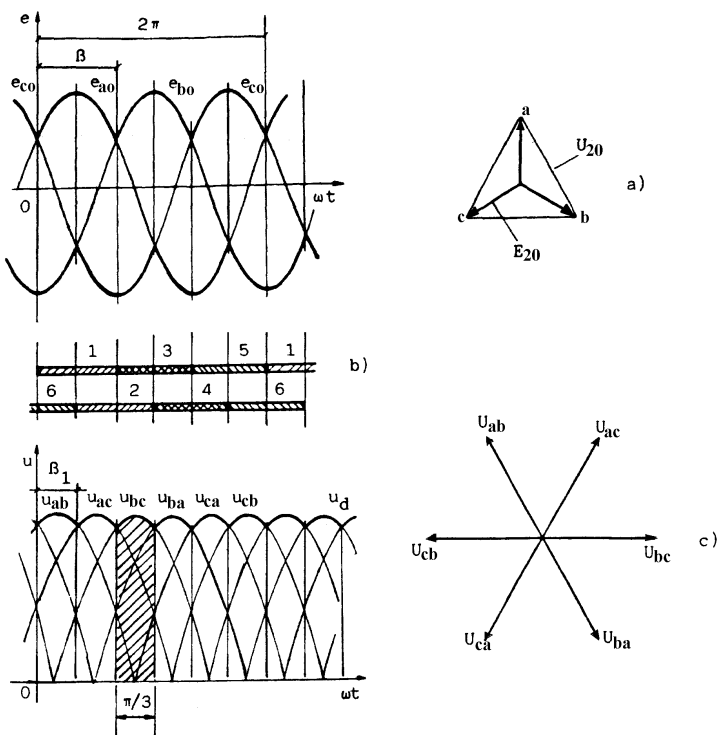

Fig.3. Diagrams of three-phase bridge

a) diagrams of phase voltages; b) conduction intervals of diodes; c) rectified voltage $\mathrm{u}_{\mathrm{d}}(\omega \mathrm{t})$ and line voltage phaosrs

$$
u_{d}(\omega t)=u_{d} \approx u_{b c}=\sqrt{2} U_{20} \cdot \cos \omega t
$$

is determined (according to the first theorem of average) with the formula:

$$
\begin{gathered}
U_{d 0}=\frac{1}{\beta_{1}} \int_{-\beta_{1} / 2}^{+\beta_{1} / 2} u_{d}(\omega t) \cdot d(\omega t)=\frac{1}{\beta_{1}} \int_{-\beta_{1} / 2}^{+\beta_{1} / 2} \sqrt{2} U_{20} \cdot \cos \omega t \cdot d(\omega t)= \\
=\frac{\sqrt{2} U_{20}}{\beta_{1}}(\sin \omega t){ }_{-\beta_{1} / 2}^{+\beta_{1} / 2}=\sqrt{2} \cdot U_{20} \cdot \frac{\sin \beta_{1} / 2}{\beta_{1} / 2}
\end{gathered}
$$

Concretely, for $\beta_{1}=\pi / 3$ the average value $\mathrm{U}_{\mathrm{d} 0}$ (of the rectified voltage $u_{d}$ ) becomes:

$$
U_{d 0}=\sqrt{2} U_{20} \cdot \frac{\sin \frac{\pi}{6}}{\frac{\pi}{6}}=\frac{3 \sqrt{2}}{\pi} \cdot U_{20} \approx 1,35 \cdot U_{20}
$$

\subsection{Diagrams of currents}

As previously assumed, the inductance $\mathrm{L}_{\mathrm{d}}$ (of the DC circuit) can be considered as infinitely high $\left(\mathrm{L}_{\mathrm{d}} \rightarrow \infty\right)$. 
Consequently the DC current $i_{d}$ will be perfectly smooth, and constant over time $i_{d}=I_{d}$. Subsequently, under these assumptions will be determined the diagrams of currents.

\subsubsection{Currents through diodes}

If the diode switching phenomenon (in each switching group) is neglected, it can be admitted that through each semiconductor diode (of the three-phase bridge) will flow the constant current:

$$
i_{D}=i_{d}=I_{d}
$$

during each conduction interval $\beta=2 \pi / 3$ of each variation period $\omega \mathrm{T}=2 \pi$ of the supply voltage.

Outside of the conduction interval, the current through the respective diode is null $\left(\mathrm{i}_{\mathrm{D}}=0\right)$.

Taking into consideration the sequence of the conduction intervals (see Fig.3, pos.b)), in Fig. 4 there are depicted (through ,rectangular blocks") the currents $i_{D 1}, \quad i_{D 3}$ şi $i_{D 5}$, and respectively $i_{D 2}, \quad i_{D 4}$ şi $i_{D 6}$ corresponding to the valves (diodes) of the two switching groups of the three-phase bridge.

The average value (on a period interval $\omega \mathrm{T}=2 \pi$ ) of the currents through the three-phase bridge is calculated with the formula:

$$
I_{\text {Dmed }}=\frac{1}{2 \pi} \int_{0}^{2 \pi} i_{D} \cdot d(\omega t)=\frac{1}{2 \pi} \int_{0}^{2 \pi / 3} I_{d} \cdot d(\omega t)=\frac{I d}{3}
$$

The effective value of the currents through the diodes $I_{D}$ is given by:

$$
I D=\sqrt{\frac{1}{2 \pi} \int_{0}^{2 \pi} i_{D}^{2} \cdot d(\omega t)}=\sqrt{\frac{1}{2 \pi} \int_{0}^{\frac{2 \pi}{3}} I_{D}^{2} \cdot d(\omega t)}=\frac{I_{d}}{\sqrt{3}}
$$

\subsubsection{Currents through secondary windings}

To the star connection (Y) of the secondary phase windings, the currents $i_{a}, i_{b}$ and $i_{c}$ in the three secondary phases of the rectifier transformer (see Fig.2) result as follows:

$$
\begin{aligned}
& \mathrm{i}_{\mathrm{a}}=\mathrm{i}_{\mathrm{D} 1}-\mathrm{i}_{\mathrm{D} 4} \\
& \mathrm{i}_{\mathrm{b}}=\mathrm{i}_{\mathrm{D} 3}-\mathrm{i}_{\mathrm{D} 6} \\
& \mathrm{i}_{\mathrm{c}}=\mathrm{i}_{\mathrm{D} 5}-\mathrm{i}_{\mathrm{D} 2}
\end{aligned}
$$

Graphically, the diagrams of secondary currents $i_{a}, i_{b}$ and $i_{c}$ depending on $\omega t$ are depicted in Fig.4. In the neglect of the switching, they are formed (on each phase) of "rectangular blocks" of amplitude $\pm \mathrm{I}_{\mathrm{d}}$ and duration $2 \pi / 3$ separated by pauses (of null value) of duration $\pi / 3$.

The average value of these alternating currents (nonsinusoidal) is null. Instead the effective value $I_{2}$ of the secondary phase currents is given by:

$I_{2}=\sqrt{\frac{1}{2 \pi} \int_{0}^{2 \pi} i_{a}^{2} \cdot d(\omega t)}=\sqrt{\frac{1}{2 \pi}\left[I_{d}^{2} \cdot \frac{2 \pi}{3}+I_{d}^{2} \cdot \frac{2 \pi}{3}\right]}=\sqrt{\frac{2}{3}} \cdot I_{d}$

\subsubsection{Currents through primary windings}

Let $i_{A}, i_{B}$ and $i_{C}$ be the three-phase system of the currents passing through the transformer primary phase windings (see Fig.2). If $\mathrm{w}_{1}$ and $\mathrm{w}_{2}$ represent the phase turns numbers of the primary and secondary, respectively from the condition of neglecting the magnetization currents in the total currents equations corresponding to each column of the core of the three-phase transformer (so in the hypothesis $\mu_{\mathrm{Fe}} \rightarrow \infty$ ) we obtain the expressions of the primary currents $i_{A}, i_{B}$ and $i_{C}$ :

$$
\begin{array}{ll}
w_{1} \cdot i_{A}+w_{2} \cdot i_{a} \approx 0 & i_{A} \approx-\frac{w_{2}}{w_{1}} \cdot i_{a} \\
w_{1} \cdot i_{B}+w_{2} \cdot i_{b} \approx 0 & i_{B} \approx-\frac{w_{2}}{w_{1}} \cdot i_{b} \\
w_{1} \cdot i_{C}+w_{2} \cdot i_{C} \approx 0 & i_{C} \approx-\frac{w_{2}}{w_{1}} \cdot i_{C}
\end{array}
$$

Consequently, the primary phase currents $i_{A}, i_{B}$ and $i_{C}$ vary (with the time) vary proportionately (being in phase opposition and having the amplitudes $\mathrm{w}_{2} / \mathrm{w}_{1}$ times increased) with the secondary phase currents $i_{a}$, $i_{b}$ and $i_{c}$. So their effective values $I_{1}$ will be proportional with $I_{2}$, meaning:

$I_{1}=\sqrt{\frac{1}{2 \pi} \int_{0}^{2 \pi} i_{A}^{2} \cdot d[\omega t]}=\frac{w_{2}}{w_{1}} \cdot I_{2}=\frac{w 2}{w_{1}} \cdot \sqrt{\frac{2}{3}} \cdot I_{d}$

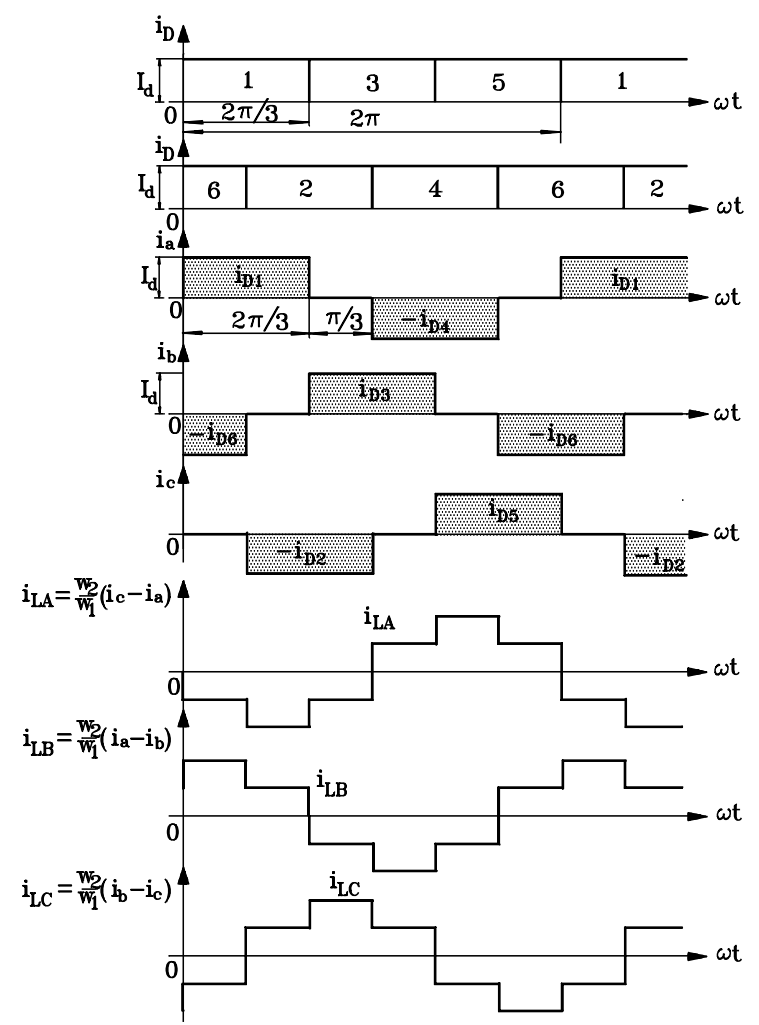

Fig.4. Diagrams of currents through diodes (iD1, $i_{D 3}$, $i_{D 5}$ and $\left.i_{D 2}, i_{D 4}, i_{D 6}\right)$, through the secondary windings $\left(i_{a}, i_{b}\right.$ and $\left.i_{c}\right)$ and the primary line currents $i_{\mathrm{LA}}, i_{\mathrm{LB}}$ and $i_{\mathrm{LC}}$

\subsubsection{Line currents in the primary}

To the delta connection $(\Delta)$ of the primary phase windings of the power transformer (see Fig.2), the three- 
phase system of the line currents $i_{L A}, i_{L B}$ and $i_{L C}$ is determined with the relationships:

$$
\begin{aligned}
& i_{L A}=i_{A}-i_{C}=\frac{w_{2}}{w_{1}} \cdot\left(i_{c}-i_{a}\right) \\
& i_{L B}=i_{B}-i_{A}=\frac{w_{2}}{w_{1}} \cdot\left(i_{a}-i_{b}\right) \\
& i_{L C}=i_{C}-i_{B}=\frac{w_{2}}{w_{1}} \cdot\left(i_{b}-i_{c}\right)
\end{aligned}
$$

Graphically, the diagrams of line currents $i_{L A}, i_{L B}$ and $i_{\mathrm{LC}}$ (depending on $\omega \mathrm{t}$ ) are depicted in the bottom side of Fig.4 [9].

The effective value $I_{1 L}$ of the line currents is given by:

$$
I_{1 L}=\sqrt{\frac{1}{2 \pi} \int_{0}^{2 \pi} i_{L A}^{2} \cdot d(\omega t)}=\sqrt{\frac{2}{2 \pi} \int_{0}^{\pi} i_{L A}^{2} \cdot d(\omega t)}=
$$

$$
=\sqrt{\frac{1}{\pi} \cdot\left(\frac{w 2}{w 1}\right)^{2}\left[\frac{\pi}{3} I_{d}^{2}+\frac{\pi}{3}\left(2 I_{d}\right)^{2}+\frac{\pi}{3} I_{d}^{2}\right]}=\frac{w 2}{w 1} \cdot I_{d} \cdot \sqrt{2}
$$

One can highlight that although the line and phase voltages vary sinusoid over time, both primary currents and secondary currents (phase and line) vary nonsinusoid over time. This way results explicitly in the deforming (non-sinusoidal) regime in which the rectifier transformer of traction substation is operating.

\section{Discussion and Conclusion}

The transmission and distribution of electricity through different voltage levels are possible due to the use of power transformers. The efficiency and sustainability of power transformers are in correlation with the reliability of the whole network, and could have considerable economic and environmental impact. Forecast based on mathematical models enlarges our beliefs on the world functionality. Although the mathematical modeling is a complex process and entails a large element of compromise the interacting systems in the real world can be studied identifying the most important interrelations of the systems. Since the efficiency standards can be expressed in terms of electrical efficiency, in order to enhance the transformer efficiency, in this study it is carried out the three-phase power transformer modelling with space phasors. The equations system obtained with space phasors can be used to analyze the dynamic regimes of three-phase transformers, being successfully applied, for instance, in the method of structural diagrams for the power transformer operation. Subsequently we have analyzed some aspects of threephase power transformer in a $\mathrm{AC} / \mathrm{DC}$ traction substation, concluding that although the line and phase voltages vary sinusoid over time, both primary currents and secondary phase and line currents vary non-sinusoid over time. This way results explicitly in the deforming (non-sinusoidal) regime in which the rectifier transformer of traction substation is operating. Looking forward the authors of this study intend to analyze the currents' harmonics in the three-phase power transformer of a $\mathrm{AC} / \mathrm{DC}$ traction substation.

\section{References}

1. M. Jufer , Électromecanique, Traité d'Électricité, Vol.IX, Presses Polytechniques Romandes, CH1015, Lausanne (1992).

2. R. Godina, E.M.G. Rodrigues, J.C.O. Matias, J.P.S. Catalao, Energies, 2015, 8, 12147-12186 (2015).

3. D.A. Nicola, C.A. Bulucea, Electrotehnica, masini si echipamente electrice (Electrotechnics, Electrical Machines and Equipment), SITECH Publishing House, Craiova, Romania (2005).

4. C.A. Bulucea, D.A. Nicola,. Introducere $\hat{\imath}$. electrotehnica si echipamente electrice (Introduction to Electrotechnics and Electrical Equipment), SITECH Publishing House, Craiova, Romania (2004).

5. G. Marion, An Introduction to Mathematical Modelling (2008), Given by Daniel Lawson and

Glenn Marion, Available at :

https://people.maths.bris.ac.uk/ madjl/course text.p df, Accessed on 15 March 2019.

6. M. Fogiel M., The Electric Circuits. Problem Solver. A complete Solution Guide to Any Textbook, Research \& Education Association (REA) Piscataway, New Jersey, SUA (1998).

7. I. Coulon, M. Jufer, Introduction à l'électrotechnique (Introduction to Electrotechnics), 5th ed., Piesses Politechniques Romandes, Lausanne (1989).

8. C.A. Bulucea, D.A. Nicola, N.E. Mastorakis, D.C. Cismaru, Recent Advances in Energy and Environment, Proc. 5th IASME/WSEAS Int.Conf. on Energy\&Environment, University of Cambridge, Cambridge, pp.234-241 (2010).

9. D.A. Nicola, Tractiune Electrica (Electric traction), Manual universitar pentru învăţământ cu frecvenţă redusă (University Book for education with brief student frequency), Editura Universitaria Craiova (Universitaria Publishing House) (2012).

10. F. Perticaroli., Sistemi elettrici per i trasporti.Trazione elettrica, Casa Editrice Ambrosiana, Milano (2001)

11. I. Cantemir, M.,Oprişor,,Tracţiune electrică (Electric traction), EDP, Bucureşti (1971) 